\title{
Modeling Nanowire Indentation Test With Adhesion Effect
}

Because of the large aspect ratio of its length to radius and the large surface area to volume ratio, the nanowire is highly flexural and susceptible to the adhesion influence. The bending deflection of nanowire and its adhesion effect make the previous indentation models inappropriate for the nanowire indentation test. In this paper, a new model incorporating the nanowire bending deflection, loading symmetry/asymmetry, and adhesion effect is presented and compared with the previous models. Because of the bending deflection of the flexural nanowire, the nanowire may lift-off/separate from its contacting elastic medium and the localized contact effects may thus be induced. The localized contact effects as predicted by this new model can cause the relatively large deflection difference of the nanowire in test as compared with those obtained by the previous models, which impacts directly and significantly on the interpretation of the indentation experimental data. The nanowire is modeled as a cylinder/beam and the indentation force is modeled as a concentrated force. The elastic medium is modeled as an elastic foundation. The elastic foundation behaves as a linear spring in nonadhesive Hertz contact and as a nonlinear softening spring in adhesive contact. In the Hertz contact, due to lift-off, the contact length is independent of the load. However, in adhesive contact, larger load results in smaller contact length. Unlike the Hertz contact in which lift-off always occurs when adhesion force is too large for bending cylinder to overcome, there is no lift-off for cylinder and the full contact scenario is thus formed.

[DOI: 10.1115/1.4002305]

\section{Introduction}

Due to their wide potential applications in small devices and remarkable physical properties, which range across the different scientific disciplines, nanowires have attracted much interest in scientific community $[1,2]$. Because of their small size and thus large surface area to volume ratio, nanowires can have some physical properties significantly different from the bulk ones. Unlike the resonance test [3] and three-point bending test [4,5] of nanowires/nanotubes, which essentially only offer the elastic modulus information, more information such as Young's modulus, hardness, yield stress, and work-hardening exponent can be obtained from the indentation test [6,7]. Nanoindenter is also known for its excellent force/displacement resolution and force control, which gives the nanoindentation great advantages in the mechanical properties test of nanomaterials [2]. However, the model for the nanowire indentation is extremely few [2]. The so-called onedimensional nanostructure [8], such as nanotube, nanobelt, and nanowire has very large aspect ratio of length to radius, which makes the structure highly flexural. The standard Oliver-Pharr method [6] assumes the indented sample as a monolithic, semiinfinite elastic half-space [7]. Due to its flexurality, nanowire in indentation test will have the nonuniformity along the axis [9], which leads to the localized effect and the separation of nanowire from its contacting substrate. The bending deflection of nanowire is the major reason that makes the Oliver-Pharr method inappropriate for modeling nanowire indentation test [2]. Yu et al. [10] developed a model for the carbon nanotube contact under indentation using the Hertz contact model and ignoring the nanotube flexurality. Feng et al. [2] reviewed several receding contact models, which can be potentially used as the model for the nanowire indentation test. Those receding contact models as discussed later in details do not incorporate adhesion effect and assume the symmetric loading scenario.

Contributed by the Applied Mechanics Division of ASME for publication in the JouRnal of Applied Mechanics. Manuscript received November 2, 2009; final manuscript received February 7, 2010; accepted manuscript posted August 3, 2010; published online October 12, 2010. Assoc. Editor: Yonggang Huang.
As shown in Fig. 1(a), a flexural cylinder is in contact with an elastic medium. The flexural cylinder can separate, or, say, lift-off from the other contacting elastic medium because of its bending deflection. In contact mechanics, the flexural structure contact problem is often referred to as the receding contact $[11,12]$, unbonded contact [13-15], and tensionless contact [16,17]. The name of receding contact $[11,12]$ emphasizes the fact that when the flexural structure is loaded, its contact area is smaller than the unloaded one due to the lift-off mechanism. Because the flexural layer of the beam/plate structure is free to lift-off, this kind of contact is thus often called the unbonded contact [13-15]. Because in nonadhesive Hertz contact the tensile stress cannot be transmitted to the lifting-off structure portions and only the compressive stress can be transmitted through the contact interface [18], this kind of contact is also often called the tensionless contact $[16,17]$. Although the calculated contact lengths $\left(x_{1}+x_{2}\right.$ as shown in Fig. 1(b)) are different due to the their modeling difference and approximate nature, all these above receding/unbonded/ tensionless contact models [13-18] reach the same conclusion that the contact length is independent on the load magnitude. Increasing load just pushes the flexural structure deeper into the elastic medium but remains the same contact length, which implies the following facts: the displacement, stress, and strain are in direct proportion to the load; however, the contact area is not [12]. Therefore, with the increasing load the elastic energy will be stored faster than the adhesion energy, which is proportional to the contact area. This will have significant impact on classical Amonton's law in tribology, which predicts the friction increases linearly with the normal pressure. Yang et al. [19] recently demonstrated such violation of Amonton's law in the contact of nanotube rafts. Amonton's law can only be valid when the contact area increases substantially faster than the stored elastic energy [19]. However, because the nanotube's Young modulus in the radial direction is relatively small compared with its Young's modulus in the axial direction, which leads to relatively large cross section deformation, the work done by the normal pressure is transformed more into the elastic energy rather than to increase the contact area/adhesion energy [19], which leads to the abnormal tribologi- 


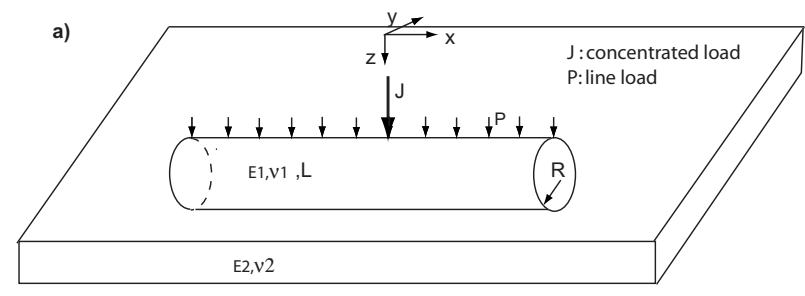

b) Contact Profile

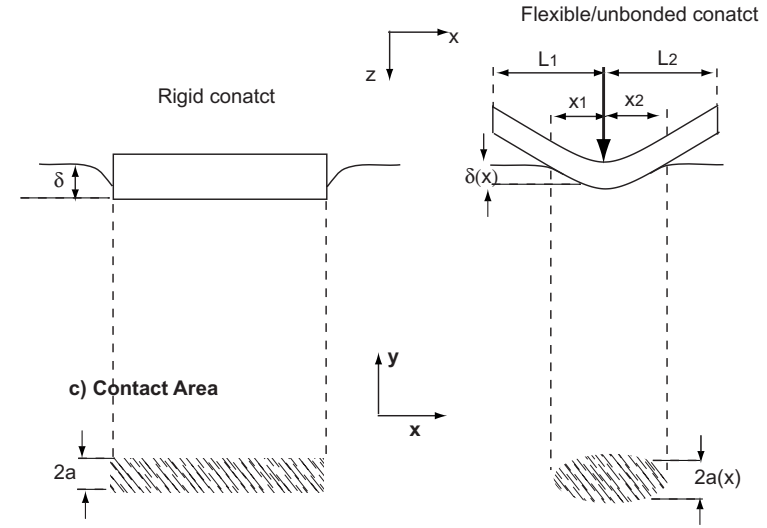

Fig. 1 (a) The schematic diagram of a cylinder with Young's modulus $E_{1}$, Poisson ratio $\nu_{1}$, radius $R$ and length $L$ in contact with an elastic medium with $E_{2}$ and $\nu_{2} . J$ is the concentrated load and $\boldsymbol{P}$ is the uniformly distributed line load. (b) The contact profile in $x-z$ plane. For the rigid contact scenario, the whole cylinder sinks into the elastic medium with a constant $\delta$. For the flexible contact scenario, the cylinder lifts-off and $\delta$ varies with $x . x_{1}$ and $x_{2}$ are the left-side and right-side contact lengths. (c) The contact area in $x-y$ plane. For the rigid contact scenario, the contact area is a rectangle and the contact width $2 a$ is constant. For the flexible contact scenario, the contact is an ellipselike zone and the contact width $2 a$ varies with $x$.

cal behavior. The flexural rigidity of the contacting layer also has the very important influence on the peeling test of adhesion measurement [20] and the instability pattern formation at the contact line $[21,22]$.

The previous models [13-18] consider the flexural rigidity of a slender layer and its interaction with the contacting elastic medium. However, in those models the adhesion effect is not taken into account. When the size of contact layer is of the micron order or smaller, the adhesion force can be a dominant one [23]. This is particularly true for those nanowire [4] and nanotube [5] in contact with the porous materials and adhesion is (assumed) so strong that the clamped-clamped boundary conditions are formed $[4,5,8]$. In the adhesive contact models for a cylinder developed by Chaudhury et al. [24], Chen and Gao [25] did not consider the effect of the cylinder flexural rigidity. In their models [24,25], the elastic deformation concentrates around the contact interface and the cylinder does not bend; therefore one cross section deformation can stand for the whole cylinder. Here, we call this kind of contact "rigid contact," which says the cylinder flexural rigidity is so large that the cylinder does not bend during contact, as shown in Fig. 1(b). The adhesive contact model developed by Ghatak et al. [20-22] focuses on the influence of the confinement/ incompressibility of the flexural film on the formation of wavy contact lines and adhesion measurement. Also in their models [20-22] the substrate is (assumed) rigid and the elastic energy is thus all stored in the flexural film; the only interaction between film and substrate is adhesion. In this paper, we incorporate the cylinder bending energy, the elastic energy due to contact, and the adhesion energy together to study the adhesive contact of a flexural cylinder.

There are two types of problem formulations on this flexural contact problem: integral and differential ones. When the elastic medium is modeled as an elastic half-space $[13-15,17,18]$, the formulation is always the integral one. The integral equation of the elastic half-space model, which indicates the displacement, strain, and stress at a point, are determined by the elastic deformation all over the area. In contrast to this, the elastic foundation model, for example, the Winkler foundation model, assumes the elastic medium consists of a series of independent springs; therefore, in an elastic foundation, the displacement, strain, and stress at a point are locally determined. The elastic foundation model leads to the differential formulation of this contact problem $[16,26,27]$. In the viewpoint of continuum approach, the elastic foundation model may lead to some physically unrealistic results, especially on the stress analysis. But Kerr [28] pointed out that the elastic foundation model is introduced to study the foundation surface response, not the stress caused inside the foundation. This deficiency of elastic foundation model on the stress analysis in general should not cause serious problem. Elastic foundation model mathematically is much simpler than the elasticity approach of the elastic half-space model [28]. The integral formulation in general is much more complex and lengthy than the differential one. Furthermore, the relationship of line loaddisplacement for the 2D (cylinder) plane-strain contact is indeterminate in an elastic half-space model [29] due to some uncertainties of its 2D elasticity features [30]. In this paper, the Winkler elastic foundation model is applied to derive the equilibrium equation. Our Winkler foundation model is developed by choosing the cylinder center displacement as the representative displacement for the whole cylinder cross section and requiring the force due to the elastic foundation to be equal to that derived by the elastic half-space model.

\section{Model Development}

2.1 Hertz Contact. Figure $1(a)$ shows a cylinder with Young's modulus $E_{1}$, Poisson ratio $\nu_{1}$, radius $R$, and length $L$ in contact with an elastic medium. The elastic medium is with Young's modulus $E_{2}$ and Poisson ratio $\nu_{2}$, which here is modeled as an elastic half-space [12] to derive all our following results. Here, the coordinate system is also shown in Fig. 1(a), which starts at the location of the concentrated load $J . P$ is the uniformly distributed line load. Figure $1(b)$ shows the scenarios of the rigid and flexible contacts in the $x-z$ plane. Here, the word rigid contact means the cylinder bending stiffness is very large and during contact the cylinder sinks into the elastic medium as a whole, i.e., the cylinder center displacement $\delta$ is a constant. For the flexible contact, there is a phenomenon called lift-off $[15,16]$, which is shown in Fig. 1(b), in which the cylinder separates from the elastic medium at the left side of $x_{1}$ and the right side of $x_{2}$. Generally, $x_{1} \neq x_{2}$ when the concentrated load $J$ is not located at the center, i.e., $L_{1} \neq L_{2}$. Figure $1(c)$ shows the contact area in $x-y$ plane. The rigid contact has a rectangular contact area, in which the half contact width $a$ is a constant. The contact area of the flexible contact is an ellipselike shape, in which $a$ varies at the different locations. It is noticed that for the rigid contact, because the cylinder submerges as a whole into the elastic medium, the total contact length is the cylinder length $L$. However, for the flexible contact, the total contact length is $x_{1}+x_{2}$ due to the lift-off mechanism. Obviously, the reason for such difference is that in the rigid contact modeling the overall flexurality/bending stiffness of the cylinder is not taken into consideration $[12,24]$.

In order to have a comparison with the previous research results $[16,26]$, physically we set the line load $P=0$ and only keep $J$ nonzero. However, the rigid contact models $[12,24]$ so far can only handle the line load case. In the modeling sense, the concen- 
(a) Geometric Relations between $\mathbf{R}$, a and $\delta$

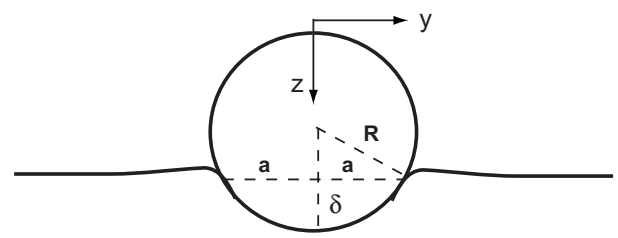

(b) Discontinuous Contact Scenario

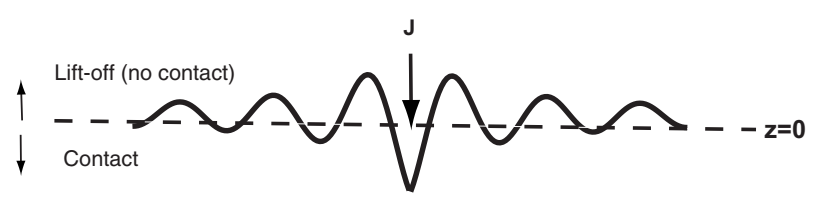

Fig. 2 (a) Geometric relations of $R, \delta$, and $a$ in a cylinder contact and $(b)$ the discontinuous contact scenario in which the cylinder has multiple separated contact zones

trated load $J$ is transformed into the line load as $P=J / L$ [24], which as shown later is a major drawback of the rigid contact modeling because it implies that the location of the concentrated load $J$ has no influence on the contact. Now let us first derive $P_{e}$, the force per unit length of the cylinder exerted by the elastic medium. As the elastic medium is modeled as an elastic halfspace and for the time being adhesion is ignored, $P_{e}$ is given as follows [12]:

$$
P_{e}=\frac{\pi a^{2} E^{*}}{4 R}
$$

Here, $E^{*}$ is the reduced Young modulus defined as $1 / E^{*}=(1$ $\left.-\nu_{1}^{2}\right) / E_{1}+\left(1-\nu_{2}^{2}\right) / E_{2}$. As shown in Fig. 2(a), the following relation holds when $a / R$ is small [12]:

$$
\delta=\frac{a^{2}}{2 R}
$$

The elasticity analysis shows that Eq. (2) is valid for the twodimensional cylinder contact only when the elastic medium is treated as an elastic foundation and thus, the surface displacement outside contact area is neglected [12]. It is also interesting to notice that the $\delta$ value is one-half of that obtained by Hertz for sphere contact $\left(\delta=a^{2} / R\right)$ [12]. As this $\delta$ is also the cylinder center displacement $(w)[25,30]$, from now on, we use $w$ instead of $\delta$ to stand for it. From Eq. (1), we have

$$
P_{e}=\frac{\pi a^{2} E^{*}}{4 R}=\frac{\pi E^{*}}{2} w=k_{1} w
$$

So $k_{1}$, the elastic foundation modulus is $k_{1}=\pi E^{*} / 2$. It should be emphasized that here we use the cylinder center displacement to stand for the whole cylinder cross section displacement and then derive this foundation modulus. Because the cross section displacement $u_{z}$ varies with $y$ as $u_{z}(y)=\left(a^{2}-y^{2}\right) / 2 R$ (here $y^{2} / 2 R$ is the parabolic approximation for the cylinder surface profile), Johnson [12] derived the foundation modulus $k_{1}$ through the following equation:

$$
P_{e}=\frac{\pi a^{2} E^{*}}{4 R}=\int_{-a}^{a} \frac{k_{1}}{h}\left(\frac{a^{2}-y^{2}}{2 R}\right) d y
$$

where $h$ is the thickness of the elastic medium. $k_{1} / h\left(a^{2}-y^{2} / 2 R\right)$ is the pressure generated inside contact zone by assuming that the elastic medium is a Winkler foundation. $k_{1}$ is derived from the above equation as $k_{1}=1.18 E^{*} h / a=1.18 E^{*} h / \sqrt{2 R w}$ [12]. So this foundation modulus $k_{1}$ derived by Johnson depends on the half contact width $a$ (or the center displacement $w$ ), which changes with the load. So the elastic foundation derived by Johnson's method of integrating the pressure all over the contact cross section is a nonlinear one, which can make the computation much more complicated. The (physical) reason causing this nonlinear elastic foundation modulus in Johnson's derivation is that the cross section displacement $u_{z}(y)=\left(a^{2}-y^{2}\right) / 2 R$ is parabolic (so is the pressure of the Winkler foundation); however, the pressure distribution derived by the half-space model is elliptic $(p(y)$ $\left.=2 P / \pi a^{2}\left(a^{2}-y^{2}\right)^{1 / 2}\right)[12]$. In our derivation, $P_{e}=\pi a^{2} E^{*} / 4 R$ is derived from the elastic half-space model and in our Winkler foundation model, we assume the pressure is proportional to the cylinder center displacement. Both our derivation and Johnson's of the elastic foundation modulus require that the line load of the cylinder derived by the elastic foundation model is equal to that obtained from the elastic half-space model. Because of the approximate nature of the elastic foundation, the elastic foundation modulus is dependent on the contacting bodies' geometry, the loading scenario, and the equivalence principle applied. For example, the elastic foundation modulus for the axisymmetric contact of sphere $\left(k_{1}=1.7 E^{*} h / a\right)$ is derived by equating the concentrated loads derived by the elastic foundation model and elastic half-space model [12], which is noticed to have a $44 \%$ difference with the above value of a cylinder; $k_{1}=0.71 E_{2}\left(E_{2} b^{4} / E_{1} I\right)^{1 / 3}(b$ : width) is derived for an infinitely long prismatic beam resting on an elastic medium under a sinusoidal load by equating the maximum moments derived by the elastic foundation model and elastic half-space model [31]. In essence, Eqs. (3) and (4) use the elastic foundation model to establish an analytical and simple relation for the line load $P_{e}$ and displacement $w$. For two-dimensional (cylinderlike) plane-strain contact, no analytical line load-displacement relation can be derived from the half-space model [30]. Some integral relations derived from the half-space model are shown to be divergent with the increase of cylinder length, which cause significant confusion [30]. Giannakopoulos [29] stated that for the plane-strain wedge indentation, the line load-displacement relation was indeterminate. An explanation was offered by Yang and Cheng [30] through their in-depth analysis on the half-space model of two dimensional contact: "there is always uncertainty on the surface deformation of the elastic half-plane, which is associated with the reference zero-point due to the feature of $2 \mathrm{D}$ elasticity."

For the rigid contact, the equation of equilibrium is obtained by setting $P=P_{e}[12,24]$

$$
P=\frac{J}{L}=P_{e}=\frac{\pi a^{2} E^{*}}{4 R}
$$

For the flexible contact, as shown in Fig. 1(b), the displacement is divided into the following three zones due to the lift-off mechanism $[26,27]$ :

$$
\begin{gathered}
E_{1} I \frac{d^{4} w_{1}}{d x^{4}}=0, \quad w_{1}<0, \quad-L_{1}<x<-x_{1} \\
E_{1} I \frac{d^{4} w_{2}}{d x^{4}}+k_{1} w_{2}=J \delta_{D}(x), \quad w_{2} \geq 0, \quad-x_{1} \leq x \leq x_{2} \\
E_{1} I \frac{d^{4} w_{3}}{d x^{4}}=0, \quad w_{3}<0, \quad x_{2}<x<L_{2}
\end{gathered}
$$

where $I$ is the cylinder area moment of inertia defined as $I$ $=\pi R^{4} / 4 .-x_{1}$ and $x_{2}$ are the points at which the cylinder lifts-off from the elastic medium. $w_{2}$ is the cylinder displacement in the contact zone; $w_{1}$ and $w_{3}$ are the displacements of the noncontact zones. For very chunky cylinder, we can imagine that the cylinder does not bend at all, which is to say $w_{i} \neq 0$ and $w_{i x}=w_{i x x}=w_{i x x x}$ $=w_{\text {ixxxx }}=0(i=1,2,3)$. Physically, $w_{i}$ now is the rigid body dis- 
placement and $w=w_{1}=w_{2}=w_{3}$. It is thus valid to transform the concentrated load into line load for chunky cylinder (i.e., $k_{1} w_{2}$ $\left.=k_{1} w=J \delta_{D}(x)=J / L\right)$. So Eq. (6) recovers Eq. (5) for the chunky cylinder. $\delta_{D}$ is the Dirac delta function and the force exerted by the indenter is thus modeled as a concentrated force. Generally, the apex of an indenter is rounded and is often approximated as a sphere [2]. An elliptical contact zone is formed in the indenternanotube contact [10]. Here, the reasons to model the indenter force as a concentrated one are the following: (1) the indentercylinder contact area/length is very small compared with the whole cylinder length and the cylinder-substrate contact area/ length, (2) the cylinder deformation is not sensitive to the details of the load distribution (inside the indenter-cylinder contact zone) [2], and (3) some of our results will be compared with the previous models, which use the concentrated force modeling $[16,26]$.

In order to have a comparison with the previous results $[16,26]$, the following nondimensionalization scheme is introduced:

$$
\begin{array}{rlll}
\xi & =\beta x, \quad W_{i}=\beta w_{i}(i=1,2,3), \quad A=\beta a, \quad \xi_{1}=\beta x_{1}, \quad \xi_{2} \\
& =\beta x_{2}, \quad l_{1}=\beta L_{1}, \quad l_{2}=\beta L_{2}, \quad l=\beta L, \quad F=\frac{J}{4 \beta^{2} E_{1} I}
\end{array}
$$

$\beta$ is defined as

$$
\beta=\sqrt[4]{\frac{k_{1}}{4 E_{1} I}}=\sqrt[4]{\frac{E^{*}}{2 E_{1}}} \frac{1}{R}
$$

where $\beta$ has the unit of $\mathrm{m}^{-1}$ and the dimensionless length $l$ defined above as $l=\beta L=\sqrt[4]{E^{*} / 2 E_{1}}(L / R)$, in essence, indicates the ratio of the cylinder length to its radius. $l$ also indicates the flexurality of a cylinder.

Now Eq. (6) becomes

$$
\begin{gathered}
W_{1 \xi \xi \xi \xi}=0, \quad W_{1}<0, \quad-l_{1}<\xi<-\xi_{1} \\
\frac{1}{4} W_{2 \xi \xi \xi \xi}+W_{2}=F \delta_{D}(\xi), \quad W_{2} \geq 0, \quad-\xi_{1} \leq \xi \leq \xi_{2} \\
W_{3 \xi \xi \xi \xi}=0, \quad W_{3}<0, \quad \xi_{2}<\xi<l_{2}
\end{gathered}
$$

Here, ( ${ }_{, \xi}=d / d \xi$. The details of solving Eq. (9) are given in the Appendix. It is also worth mentioning that it is not difficult at all to solve the problem of adding a line load in Eq. (6), which is the case of considering the cylinder weight [16]. There are three reasons here for us to ignore the line load induced by the cylinder weight: (1) to compare our results with the previous models, which are developed without the line load; (2) Gladwell [14] showed that when the total weight of the beam layer is less than $1 \%$ of the total applied force, which is usually the case in the indentation test, the weight of flexural layer can be ignored; and (3) as we later consider the adhesive contact case in which the adhesion force of a micron size structure can be a million times greater than the gravity force [23].

For the rigid contact, the dimensionless displacement is derived from Eq. (5) simply as follows:

$$
W=\frac{F}{l}
$$

As for the dimensionless half contact width $A=\beta a$ and from geometric relation of Eq. (2), we have the following equation when the cylinder is in contact, i.e., $W>0$.

$$
\frac{A}{\sqrt{2 \beta R}}=\sqrt{W}
$$

2.2 Adhesive Contact. The following line load exerted on the cylinder by the elastic medium is derived by considering the adhesion effect [24]

$$
P_{e}=\frac{\pi a^{2} E^{*}}{4 R}-\sqrt{4 E^{*} \pi \gamma a}
$$

where $\gamma$ is the surface energy per unit area of a surface. Again, Eq. (12) applies only for the contact zone and the half contact width $a$ is a function of $x$. By applying the geometric relation of Eq. (2), Eq. (12) is now written as follows:

$$
P_{e}=k_{1} w-k_{2} w^{1 / 4}
$$

where $k_{2}$ is defined as $k_{2}=2 \sqrt[4]{2 R\left(\pi E^{*} \gamma\right)^{2}}$. Clearly by considering the adhesion effect, the elastic foundation model of the elastic medium becomes nonlinear. Clearly with the adhesion effect, the restoring line load of Eq. (13) is smaller than the nonadhesive one. Therefore, the adhesive elastic foundation described by Eq. (13) will behave as a softening spring [32]. Because of this nonlinearity due to the adhesion, it is impossible for us to have the solution form as given in Eq. (A1). Here, the finite element method is applied to solve the adhesive contact.

The system energy is written as follows:

$$
\int_{-L_{1}}^{L_{2}} \frac{E_{1} I w_{x x}^{2}}{2} d x+\int_{-x_{1}}^{x_{2}}\left(\frac{k_{1} w^{2}}{2}-\frac{4}{5} k_{2} w^{5 / 4}\right) d x-\int_{-L_{1}}^{L_{2}} J \delta_{D}(x) w d x=0
$$

The first term is the bending energy of the cylinder, the second term is the energy stored by the elastic foundation $\int_{-x_{1}}^{x_{2}}\left(\left(k_{1} w^{2} / 2\right)\right.$ $\left.-(4 / 5) k_{2} w^{5 / 4}\right) d x=\int_{-x_{1}}^{x_{2}} \int_{0}^{w} P_{e} d w d x$. The third term is the work done by the concentrated load. It is worth pointing out here that in Eq. (14), the membrane energy [33-36] is not accounted. The membrane energy is due to the in-plane stretching/compression of structure, which holds a vital role in the wrinkling of hard film/ nanotube on soft substrate [33-36]. Therefore, Eq. (14) physically indicates that the nanowire is under no axial force and the deflection is relatively small. Equation (14) also assumes that the nanowire in test is in an isolated state, i.e., the interaction energy between nanowires is not accounted. When the two nanotubes are close to each other, their in-plane van der Waals force (per unit length) can be much larger than $P_{e}$ of the transverse normal force (per unit length) due to the nanotube/substrate interaction [36], which can cause the change of the buckling wavelength of nanotubes $[34,36]$. By using the nondimensionalization scheme of Eq. (7) and taking the variation, Eq. (14) now becomes as

$$
\frac{1}{4} \int_{-l_{1}}^{l_{2}} W_{\xi \xi} \delta W_{\xi \xi} d \xi+\int_{-\xi_{1}}^{\xi_{2}}\left(W-\alpha W^{1 / 4}\right) \delta W d \xi-F \int_{-l_{1}}^{l_{2}} \delta_{D}(\xi) d \xi=0
$$

Here, the dimensionless parameter $\alpha$ is defined as

$$
\alpha=\frac{k_{2}}{k_{1}} \beta^{3 / 4}=4 \sqrt[4]{2} \sqrt{\frac{\gamma}{\pi E^{*} R}}\left(\frac{E^{*}}{2 E_{1}}\right)^{3 / 16}
$$

Clearly, $\alpha \propto \sqrt{\gamma / R E^{*}}\left(\gamma\right.$ has the unit of $\mathrm{N} \mathrm{m}^{-1}$ and $E^{*}$ of $\mathrm{N} \mathrm{m}^{-2}$ ). So the adhesion effect $(\gamma)$ and cylinder size effect $(R)$ are incorporated in the parameter $\alpha$. For different materials, $\gamma / E^{*}$ is around $10^{-9}-10^{-12} \mathrm{~m}$ [37-39]. Our computations show that in general cases, only when $\alpha \geq 10^{-3}$ can this dimensionless parameter has (significant) impact on the deflection behavior of the cylinder. Physically, this means that only when the wire radius $R$ is of the micron scale or smaller can the adhesion effect $(\alpha)$ has impact on the cylinder deflection. This dimensionless parameter $\alpha$ (for cylinder) also reminds us the famous dimensionless number defined by Tabor [40] for the contacting spheres and it is thus often referred to as Tabor number. Tabor number holds the vital role of differentiating different contact models [41-43]. Tabor number is defined as $\mu=\left[R \gamma^{2} /\left(E^{* 2} z_{o}^{3}\right)\right]^{1 / 3}$ ( $R$ is the sphere radius and $z_{o}$ is the equilibrium separation of atoms). $z_{o}$ arises here because of the Lennard-Jones potential [43]. $\alpha$ here plays the same role for cyl- 


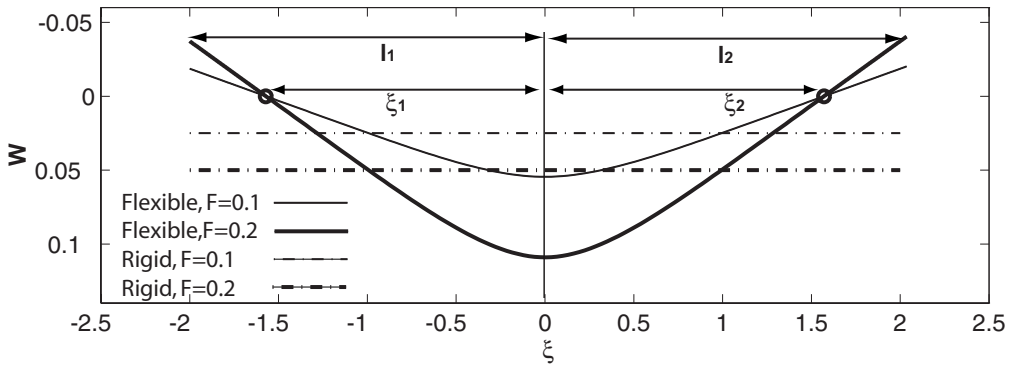

b)

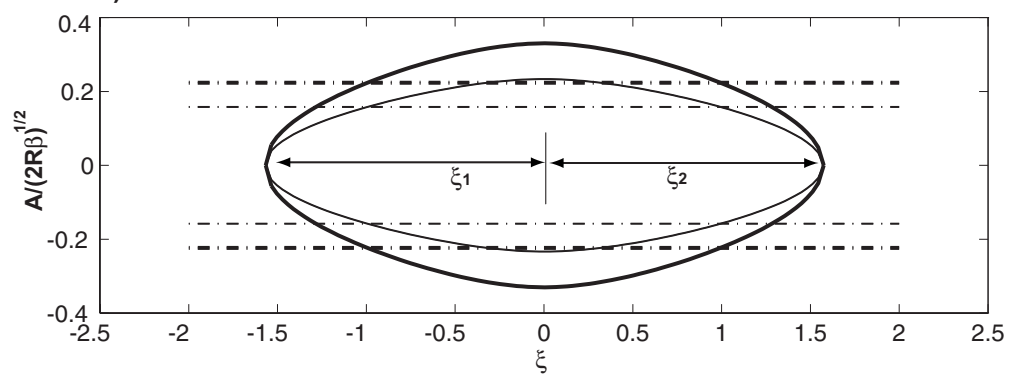

Fig. 3 (a) The displacement comparison of flexible contact and rigid contact when $l=4$ and $F=0.1,0.2$, respectively. The concentrated load $F$ is at center, i.e., $I_{1}=I_{2}=2$. (b) The comparison of contact zones.

inder as that of $\mu$ for the sphere, which is to evaluate the adhesion contribution as compared with the elastic one. One mathematic distinction is that larger $R$ results in smaller $\alpha$ but larger $\mu$.

Now in each element, we introduce the following interpolation:

$$
W=\sum_{i=1}^{4} N_{i}(\zeta) d_{i}
$$

Here, $\zeta=\xi / L_{e}$ and $L_{e}$ is the element length. $d_{1}$ and $d_{3}$ are the nodal displacements; $d_{2}$ and $d_{4}$ are the nodal rotations. $N_{i}$ are the interpolation functions defined as follows [44]:

$$
\begin{gathered}
N_{1}(\zeta)=1-3 \zeta^{2}+2 \zeta^{3}, \quad N_{2}(\zeta)=L_{e} \zeta\left(1-2 \zeta+\zeta^{2}\right), \\
N_{3}(\zeta)=\zeta^{2}(3-2 \zeta), \quad N_{4}(\zeta)=L_{e} \zeta^{2}(\zeta-1)
\end{gathered}
$$

Then substitute Eq. (17) into Eq. (15) and use the routine finite element procedures to formulate Eq. (15), a nonlinear equation set with $d_{i} s$ as unknown is obtained [44]. Here again, keep in mind that lift-off points $\xi_{1}$ and $\xi_{2}$ in Eq. (15) are unknown, too. Besides the Newton-Rhapson method [45], the steps of solving Eq. (15) are as follows: (1) first, guess the finite element nodal displacement/rotation $\left(d_{i}\right)$ and the lift-off points $\xi_{1}$ and $\xi_{2} ;(2)$ substitute them into Eq. (15) to have the finite element formulation and find the solution; (3) check the displacement solution to find out new $\xi_{1}$ and $\xi_{2}$; and (4) go back to step (2) and iterate until the displacements, $\xi_{1}$ and $\xi_{2}$, are converged. When searching the new lift-off points, we only need to seek two points $\xi_{1}$ and $\xi_{2}$, at which the left and right sides begin to sink into the elastic medium. There is a possible scenario called discontinuous contact, as shown in Fig. 2(b). But for this discontinuous contact scenario to occur, the concentrated load is so large and well beyond the material strength range [16], which makes it physically impossible to occur because the structure will be broken long before the discontinuous contact can occur. All our above derivations actually assume the linear elasticity implicitly. Standard Oliver-Pharr indentation involves large plastic deformation [6,7]. In the nanowire indentation test [10], there are two contacting interfaces: the indenter/nanowire interface and the nanowire/substrate interface, which is also often referred to as the double contact [2]. At the indenter/nanowire interface, severe plastic deformation may oc- cur. But as mentioned before, plastic deformation will change the load distribution inside the indenter/nanowire contact zone, which has little influence on the the nanowire deflection [2]. Our model actually ignores the stress distribution details inside the indenter/ nanowire contact zone and uses a concentrated load modeling. For the nanowire/substrate contact, the flexural bending transfers the concentrated load to the whole nanowire and the nanowire/ substrate contact zone is relatively large. Therefore, plastic deformation is not expected in the nanowire/substrate contact zone. The receding contact models $[11,13,14,18]$ reviewed and used by Feng et al. [2] are all elastic ones. In all finite element computations presented in this paper, the element number is fixed as 100, which already shows very high accuracy. The nonlinear equation set obtained by the finite element formulation is not sensitive to the initial guess of lift-off points and cylinder displacement. Usually, it only takes a few iterations to have the solutions converged.

\section{Results and Discussions}

3.1 Hertz Contact. Figure 3 shows (a) the displacements and (b) the contact width of the rigid and flexible contact for relatively short cylinder of $l=4$ under two different concentrated loads $F$ $=0.1$ and 0.2 . The load is symmetric, i.e., $l_{1}=l_{2}=2$. For the rigid contact, the displacement is calculated by Eq. (10). Clearly, Eq. (10) indicates the constant displacement for the whole cylinder. $W=0.1 / 4=0.025$ for $F=0.1$ and $W=0.2 / 4=0.05$ for $F=0.2$. Because the half contact width is calculated accordingly by Eq. (11) for both the rigid and flexible contacts, the constant displacements of rigid contact all over the cylinder also means that the contact width are constant, which forms the rectangular contact area. For the flexible contact, the cylinder lifts-off and the lift-off points are marked by two circles. The very counterintuitive thing about this flexible contact is that the contact length does not depend on the load magnitude $[15,16,26,27]$. As seen in Fig. 3(a), $F=0.2$ just pushes the cylinder deeper into the elastic medium than that of $F=0.1$; the two deflections share the same lift-off points and $\xi_{1}$ $=\xi_{2}=\pi / 2[16,26]$. For shorter cylinder, for example, $l<\pi$, the cylinder has less flexurality and the whole cylinder sinks into the elastic medium with the varying displacements [26]. For the flexible contact, the contact is limited to the $-\xi_{1} \leq \xi \leq \xi_{2}$ and the 
(a)
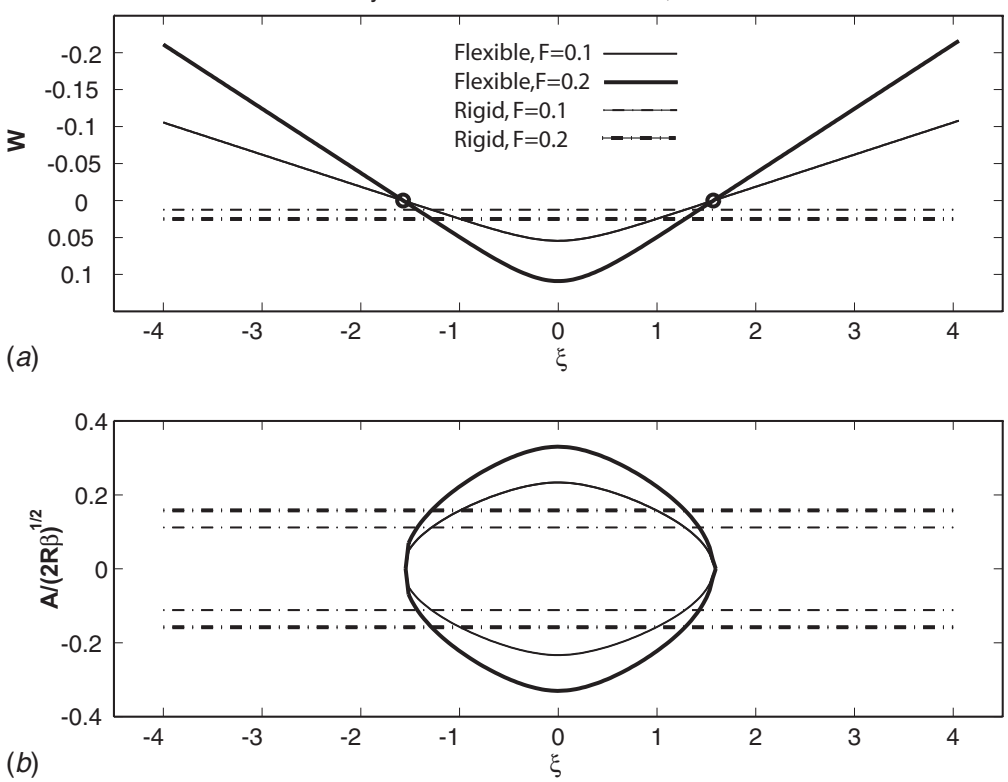

Fig. 4 (a) The displacement comparison of flexible contact and rigid contact when $I=8$ and $F=0.1,0.2$, respectively. The concentrated load $F$ is at center, i.e., $I_{1}=I_{2}=4$. (b) The comparison of contact zones.

contact area is an ellipselike area. The maximum half contact width is at $\xi=0$, at $\xi=-\xi_{1}$, and $\xi_{2}$ in which the half contact width is zero. Clearly, the contact areas of the rigid and flexible contacts are significantly different, which has a direct impact on the evaluation of the nanowire hardness (defined as the ratio of the concentrated load to the contact area) $[2,6,7]$. In this study, the dimensionless cylinder length $l$ is varied to change its flexurality. Figure 4 shows (a) the deflections and (b) the contact width of $l=8$ with the symmetric loads of $F=0.1$ and 0.2 , respectively. Interestingly, the cylinder length change has no influence on the contact length in the flexible contact and it still remains the same as $\xi_{1}+\xi_{2}=\pi$. Compared with the case of $l=4$ with the same concentrated load, the displacements of $l=8$ outside the contact area $((W(\xi), \xi<$ $-\xi_{1}$, and $\left.\xi>\xi_{2}\right)$ tilt much higher. But the displacement inside the contact area $\left(W(\xi),-\xi_{1} \leq \xi \leq \xi_{2}\right)$ remains the same. So as the result, for the flexible contact, the contact width are the same as those of $l=4$ under the same concentrated load $F$. As mentioned before, for the rigid contact, the concentrated load $F$ needs to be (simply) transformed to as the line load of $F / l$ uniformly distributed on the cylinder. Now for $l=8$, the displacement of rigid contact now become $W=0.1 / 8=0.0125$ for $F=0.1$ and $W=0.2 / 8$ $=0.025$ for $F=0.2$. Consequently, according to Eq. (11), the contact width of the rigid contact also changes. Figure 5 shows the asymmetric contact scenario and the cylinder length is $l=8$. The
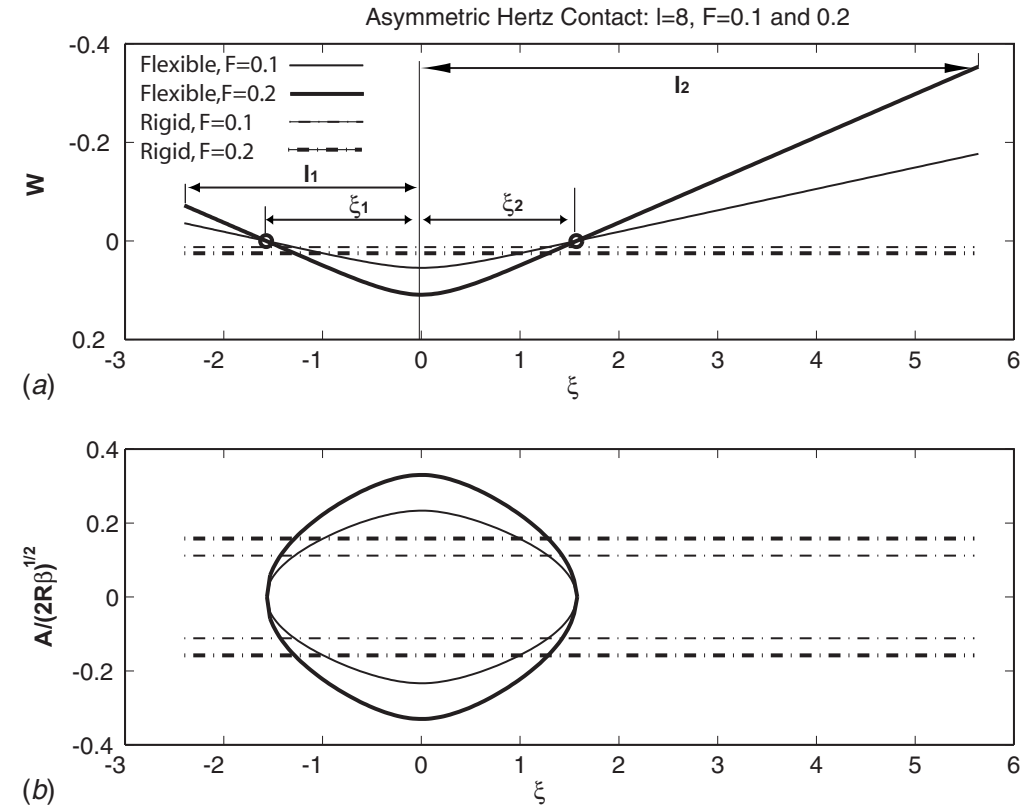

Fig. 5 The asymmetric contact scenario. The cylinder length is $I=8$ and the concentrated force $F$ is located at $l_{1}=2.4$ and $l_{2}=5.6$. The comparison of (a) the displacement and $(b)$ the contact zone. 


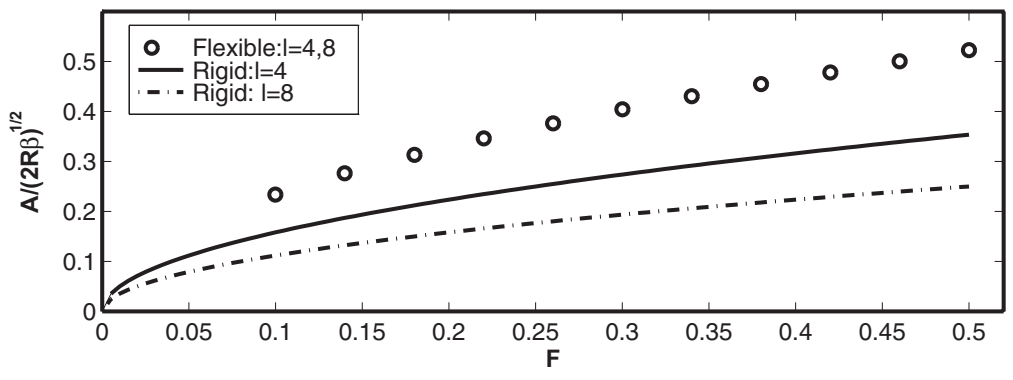

Fig. 6 (a) The comparison of $A / \sqrt{2 R \beta}$ of the rigid and flexible contact scenarios as a function of $F$ for $I=4$ and 8 , respectively. For the flexible contact, $A$ is taken at $\xi=0$. (b) The comparison of $A / \sqrt{2 R \beta}$ of the rigid and flexible contact scenarios as a function of $I$ for $F=0.1$ and 0.5 , respectively.

concentrated load $F$ is located at $l_{1}=2.4$ and $l_{2}=5.6$. Equation (10) cannot differentiate the difference between the symmetric and asymmetric loadings. Therefore, the cylinder displacements and contact width of the rigid contact in Fig. 5 are the same as those in Fig. 4. Obviously, for the deflection profiles of the asymmetric loading in Fig. 5 are quite different from those of the symmetric ones in Fig. 4. However, the contact lengths and the displacements inside the contact zone remain the same as those in Fig. 4. Therefore, symmetric and asymmetric loadings result in the same contact area in the flexible contact.

Now, let us summarize the differences between the rigid contact model and the flexible contact model here. The rigid contact model [24] transforms the concentrated load to a uniformly distributed line load and it cannot tell the difference between symmetric and asymmetric loading. With the concentrated load fixed, the cylinder length change will alter the line load magnitude; therefore, the displacement and the half contact width change accordingly. For the flexible contact of slender cylinder (for example, $l \geq 4$ ), the contact length and displacement inside the contact zone do not depend on the cylinder length $l$. The contact length is also independent of the concentrated load magnitude. Larger concentrated load just pushes the same portion of the cylinder deeper into the elastic medium, which enlarges the displacement inside the contact area and the contact width. The symmetric and asymmetric loadings do alter the overall deflections of the cylinder. But the displacement inside the contact area and the contact width remain the same for the symmetric and asymmetric loadings with the same magnitude. Figure 6 is another way of putting the above summarization in figure. Figure 6 plots the $A / \sqrt{2 \beta R}$ as a function of $F$ for $l=4$ and $l=8$ and keep in mind that $\sqrt{W}=A / \sqrt{2 \beta R}$ of Eq. (11). As for the flexible contact, $A / \sqrt{2 \beta R}$ is a function of $\xi$ and in Fig. 6, we take its maximum value, i.e., when $\xi=0$. For both the contacts, the curves keep monotonously increasing. For the flexible contact, the cylinder length $l$ has no influence on the contact width. As for the rigid contact, the longer the cylinder means the $F$ smaller line load, so the curve of $l=8$ is always below that of $l=4$.

3.2 Adhesive Contact. The adhesive contact is computed by the finite element formulation of Eq. (15). When $\alpha=0$, the results are expected to be the same of those obtained by Eq. (A1) given in the Appendix, our finite element computation results match (almost) exactly as those of Eq. (A1). One of the interesting and also counterintuitive results about the Hertz flexible contact is that the parameters, such as the loading location/symmetry, the cylinder length, and magnitude of the concentrated load have no influence on the dimensionless contact length, which is a constant of $\pi$. In this section, we will examine closely how the variation of $\alpha$ can change the contact length and displacement.

Figure 7 shows the cylinder deflections of $l=4$ and $F=0.1$ with different $\alpha=0,1 \times 10^{-3}$, and $3 \times 10^{-3}$. Here, $F$ is symmetrically loaded. $\alpha=0$ is the Hertz contact and $\xi_{1}=\xi_{2}=\pi / 2$. For $\alpha=1$ $\times 10^{-3}$ the contact length becomes larger as $\xi_{2}=1.76$ and so does the displacement inside the contact area. As for $\alpha=3 \times 10^{-3}$, the whole cylinder is now in contact with the elastic medium and is pushed deeper. As seen from Fig. 7, the cylinder bending shapes almost have no change, so the force per unit length due to the cylinder bending $\left(E_{1} I w_{x x x x}\right)$ almost has no changes, too. As indicated by Eq. (13), when considering the adhesion effect, the elastic foundation is like a softening spring. Therefore, in comparison with the Hertz contact, the adhesive contact has to increase either the contact length or the displacement inside the contact area or both to balance the same amount of external load $F$. Figure 8 examines the cylinder contact of $l=4$ and $\alpha=1 \times 10^{-3}$ under two different loads of $F=0.1$ and 0.2 , respectively. It is interesting to notice that larger $F$ has smaller contact length. $\xi_{2}=1.68$ for $F$ $=0.2$ and $\xi_{2}=1.76$ for $F=0.1$. As seen in Fig. 8, under larger $F$, the cylinder deflection is more "curvy" and the cylinder sinks much deeper into the elastic foundation, which makes the force exerted by the foundation larger and this can serve as a qualitative explanation for why larger load has smaller contact length. About the independence of the contact length on the load magnitude, we need to point out that there is no shear force inside the Winkler foundation and in the continuum modeling of elastic half-space, the shear force does exist [12]. The shear force has significant influence on this contact length [16,27]. But once the shear force contribution is specified in the Reissner foundation, the contact length is still independent on the load magnitude, which is also demonstrated by the elastic half-space models [14,15,17]. Here, it also needs to point out that there is no initial gap separating the cylinder and elastic medium. As demonstrated by Zhang and Murphy [27], larger load has larger contact length when the cylinder/ beam is initially separated with a finite gap distance from the elastic foundation. Figure 9 shows the center displacement $W(0)$ and $\xi_{2}$ as a function of $\alpha$ under $F=0.1$ and $F=0.2$, respectively. Clearly, as indicated in Fig. 9, larger $F$ always has larger $W(0)$ and smaller $\xi_{2}$, which is also shown in Fig. 8. As shown in Fig. 8, both $\xi_{2} s$ of $F=0.1$ and 0.2 starts at $\xi_{2}=\pi / 2$ at $\alpha=0$ (Hertz contact). For $F=0.1$, the whole cylinder is in contact with elastic medium at $\alpha=1.56 \times 10^{-3}$ and $F=0.2$ at $\alpha=2.64 \times 10^{-3}$. And once the full contact occurs, it keeps that way with the increase of $\alpha$.

Now, let us examine how the cylinder flexurality influences the contact. In Fig. 10 the cylinder length is $l=8$ and $F=0.1$. The concentrated force is also symmetrically loaded and $\alpha$ varies as $\alpha=0,4 \times 10^{-3}$, and $8 \times 10^{-3}$, respectively. Now, $\xi_{2}=\pi / 2$ for $\alpha$ $=0$ and $\xi_{2}=2.24$ for $4 \times 10^{-3}$. When $\alpha=8 \times 10^{-3}$, the full contact occurs. Compared with Fig. 7, under $F=0.1$, larger $\alpha$ is required for longer cylinder to have full contact. We also notice that the cylinder deflection shape has very significant change at the full contact. In Fig. 11, we examine the deflections of this longer cylinder under $F=0.1$ and 0.2 , respectively. Similarly, the curve of 


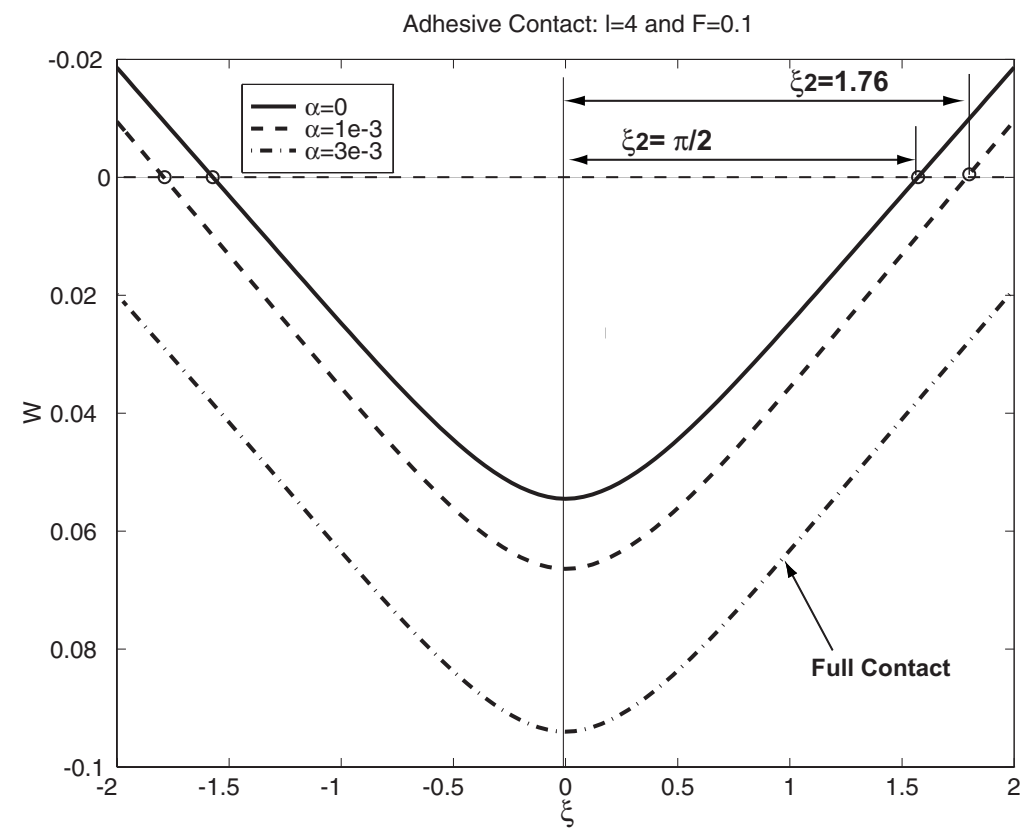

Fig. 7 The cylinder displacements of $I=4$ and $F=0.1$ for $\alpha=0,1 \times 10^{-3}$, and $3 \times 10^{-3}$, respectively

$F=0.2$ is more "curvy" resulting in a shorter $\xi_{2}=1.84$ compared with $\xi_{2}=1.84$ for $F=0.1$. Again, Fig. 12 shows the center displacement $W(0)$ and $\xi_{2}$ of this longer cylinder as a function of $\alpha$ under $F=0.1$ and $F=0.2$, which are very similar to those of Fig. 9 . The main difference is as follows: unlike that the full contact is gradually reached for shorter cylinder in Fig. 9, we see here the full contact is reached suddenly at $\alpha=4.48 \times 10^{-3}$ for $F=0.1$ and $\alpha=7.2 \times 10^{-3}$ for $F=0.2$. For $F=0.1, \xi_{2}=2.48$ at $\alpha=4.32 \times 10^{-3}$ just before its sudden jump to $\xi_{2}=4$ at $\alpha=4.32 \times 10^{-3}$; for $F$ $=0.2, \xi_{2}$ is also 2.48 at $\alpha=7.04 \times 10^{-3}$ just before its sudden jump to $\xi_{2}=4$ at $\alpha=7.2 \times 10^{-3}$. So far, we are not clear about what mechanism causes the instabilitylike jump. The analogous thing is the mode jumping phenomenon, which also occurs for a beam on a nonlinear elastic foundation [46,47]. When the axial compressive load reaches a critical value in its post-buckling region, mode jumping occurs in which the beam experiences a sudden discontinuous change in the wavelength and magnitude of its postbuckling configuration $[46,47]$.

We should talk about the contact under tension, i.e., $J<0$ case. It is well known that in the rigid contact model of adhesive contact, for example, the Johnson-Kendall-Roberts (JKR) model for the contact of spheres [12], when the load becomes zero, the spheres will not separate from each other unlike those of the Hertz contact model. Because of adhesion, the spheres can withstand the tension and they will separate only when the external tension load reaches some critical value [12], which is also true for the adhe-

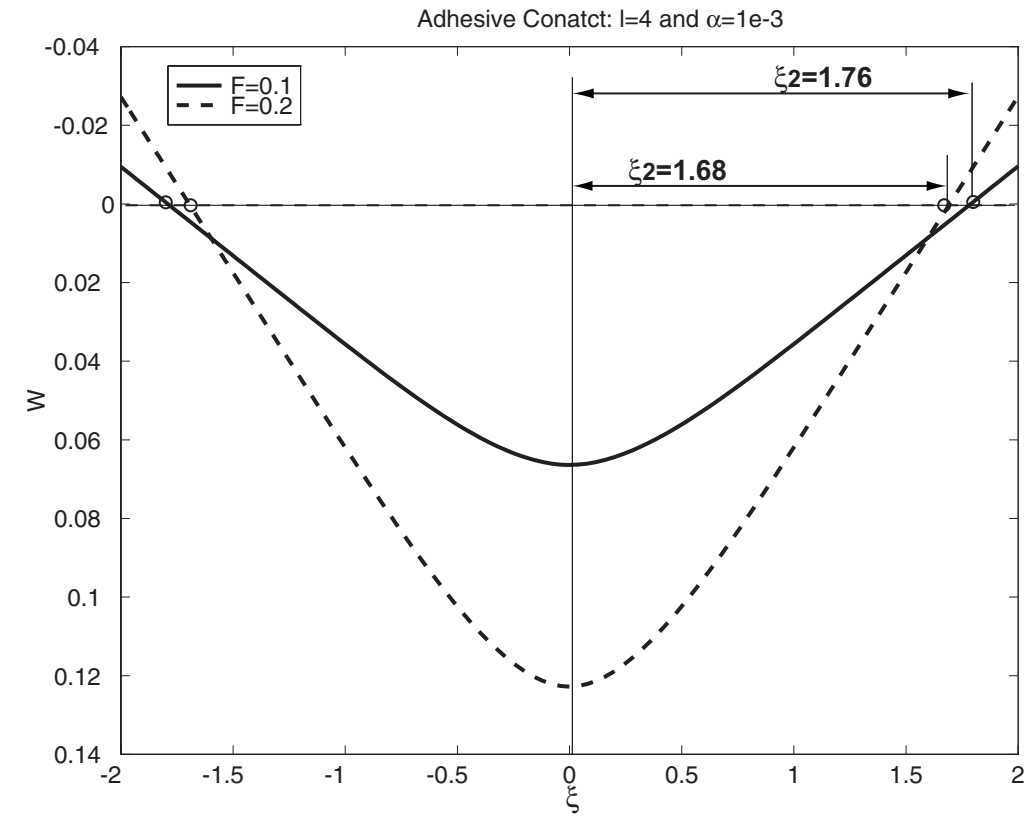

Fig. 8 The comparison of the cylinder displacements of $I=4$ and $\alpha=1$ $\times 10^{-3}$ for $F=0.1$ and 0.2 , respectively 

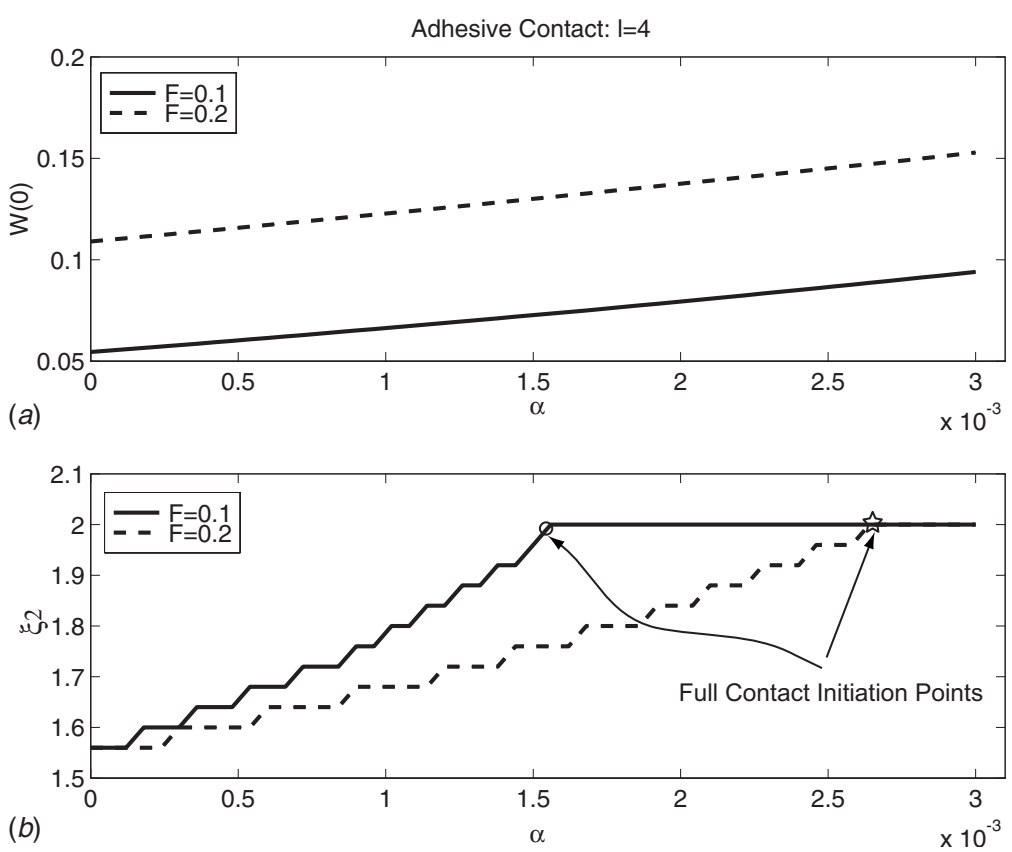

Fig. 9 (a) The cylinder center displacement $(W(0))$ of $I=4$ as a function of $\alpha$ for $F=0.1$ and 0.2 , respectively. (b) The right-side contact length $\left(\xi_{2}\right)$ as a function of $\alpha$ for $F=0.1$ and 0.2 , respectively.

sive contact of a cylinder [24]. The minimum tension required to separate the contacting bodies is called force of adhesion [12] or pull-off force [24], which has very important application in the experimental measurement of adhesion $[12,24]$. But here, it is difficult for us to define the pull-off force for the flexible contact. Unlike the rigid contact model in which the cylinder moves up and down with one displacement for the whole cylinder, the cylinder displacement of the flexible contact model is different from point to point. As seen from Fig. 13, when the load is compressive $(J>0)$, only the portion around the loading location is in contact; when the load is tensile $(J<0)$, the portion around the loading location separates from the elastic medium instantly, but the two portions around the free ends are now in contact. The only way for the cylinder of the flexible contact to be freed from contact is to further increase the tension until it pulls the whole cylinder out of the elastic medium. As seen from Figs. 3-5, the cylinder flexurality/length and loading location have significant impact on when the whole cylinder separates from the elastic medium. It will be of great trouble for the flexible contact model to measure the pull-off force to determine the adhesion.

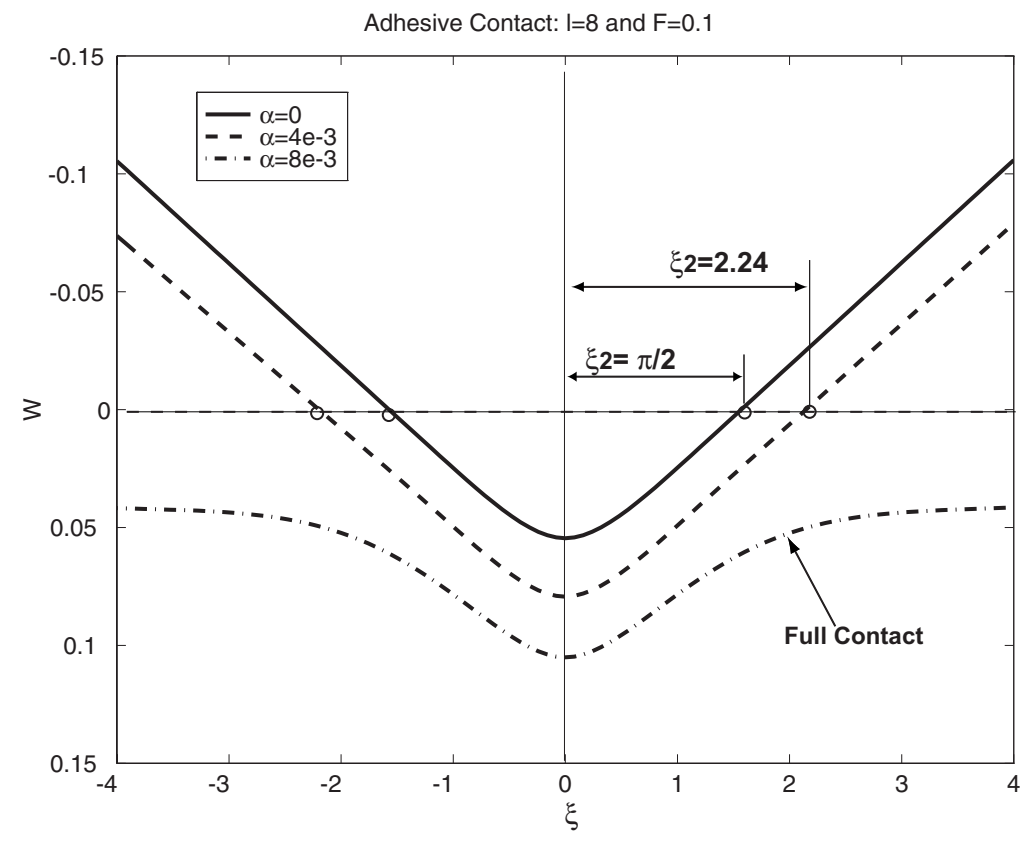

Fig. 10 The cylinder displacements of $I=8$ and $F=0.1$ for $\alpha=0,4 \times 10^{-3}$, and $8 \times 10^{-3}$, respectively 
Adhesive Contact: $I=8$ and $\alpha=4 \mathrm{e}-3$

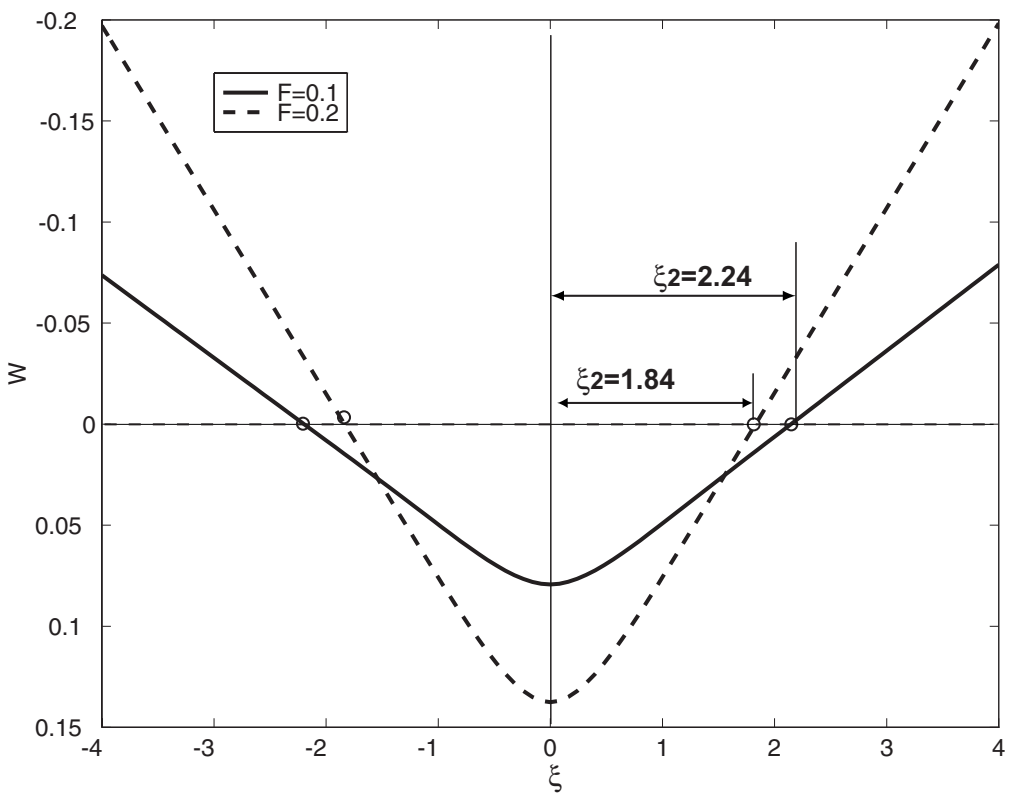

Fig. 11 The comparison of the cylinder displacements of $I=8$ and $\alpha=4$ $\times 10^{-3}$ for $F=0.1$ and 0.2 , respectively

\section{Conclusion}

In the rigid contact model, the elastic deformation occurs only around the contact interface and the structure overall stiffness is not accounted, which works fine for the structures, such as sphere and punch. However, for structure such as cylinders, especially when its ratio of length to radius is large, the structural overall stiffness/flexurality will have significant impact on the contact. Due to the structural flexurality and the compatibility conditions, the cylinder may have the lift-off phenomenon. And we show that due to this lift-off mechanism, the difference between the flexible contact and rigid contact can be very significant. In the Hertz contact of the slender cylinder, because of the lift-off mechanism, the cylinder has a constant contact length independent of the load magnitude, location, and cylinder length. The cylinder displacement and contact width depend only on the load magnitude. The adhesion together with the cylinder dimensions (radius and length) has impact on the cylinder contact length, displacement, and contact width. Under the same conditions, the adhesive contact always has the larger contact length than that of the Hertz contact. In our study, a dimensionless parameter $\alpha$ is defined and $\alpha \propto \sqrt{\gamma / R E^{*}}$, which indicates the interaction between the adhesion energy $\gamma$, contacting bodies' elastic property $E^{*}$, and the cylinder
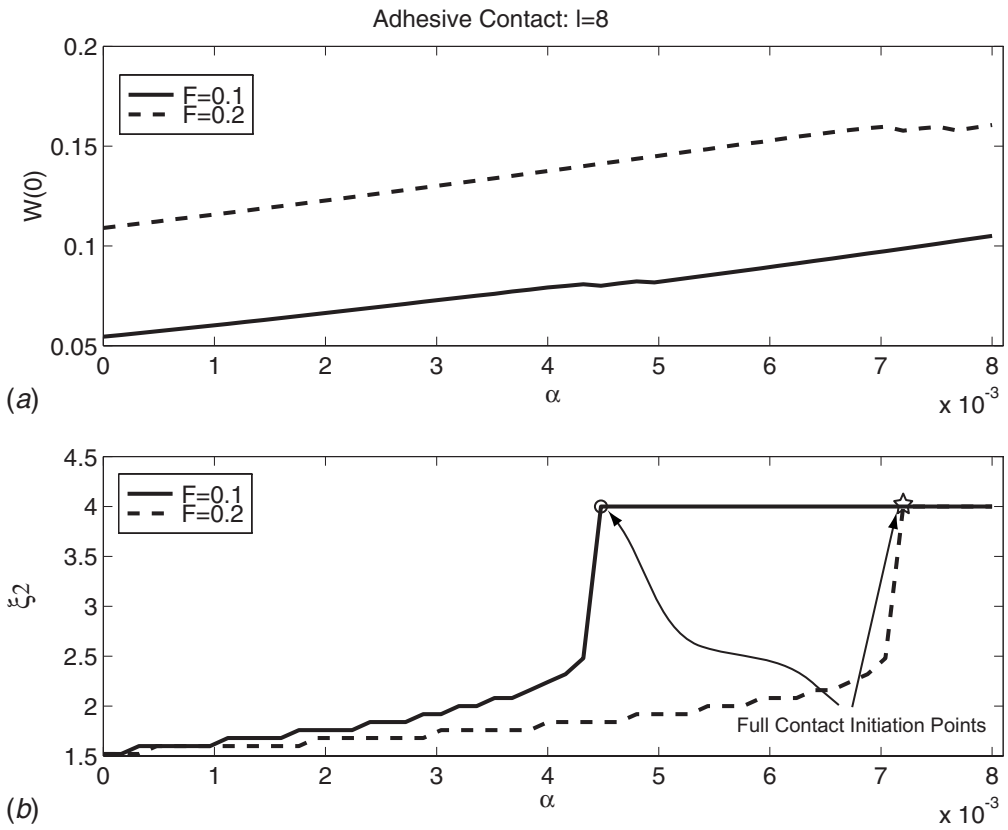

Fig. 12 (a) The cylinder center displacement $(W(0))$ of $I=8$ as a function of $\alpha$ for $F=0.1$ and 0.2 , respectively. $(b)$ The right-side contact length $\left(\xi_{2}\right)$ as a function of $\alpha$ for $F=0.1$ and 0.2 , respectively. 


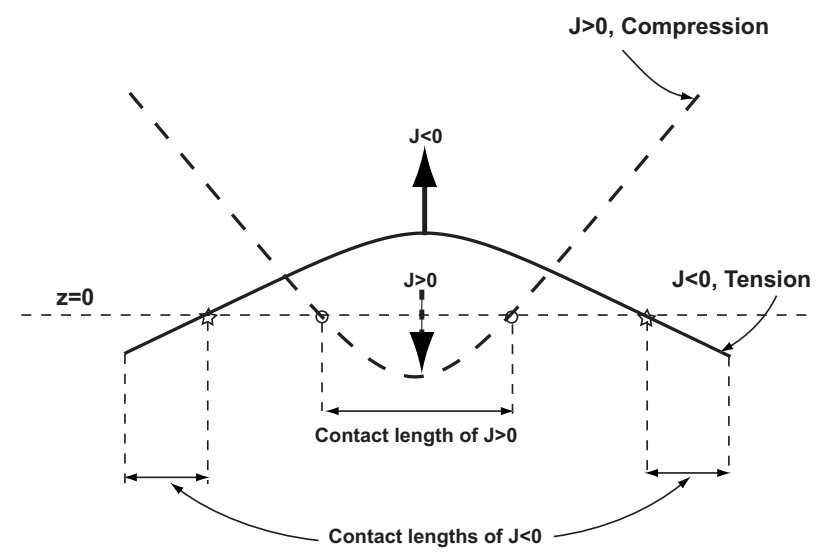

Fig. 13 Comparison of the cylinder contact scenarios under tension and compression radius $R$. Larger $\alpha$ always results in the larger contact length. When $\alpha$ is large enough, the bending cylinder is unable to overcome the adhesion force, no lift-off occurs and the full contact scenario is formed.

\section{Acknowledgment}

This work is supported by the National Natural Science Foundation of China (NSFC) (Grant Nos. 10721202 and 10772180), the LNM initial funding for young investigators, the Ministry of Science and Technology (MOST) (Grant No. 2010CB631004), and the National Basic Research Program of China (973 Program, Grant No. 2007CB310500).

\section{Appendix}

The solution forms to Eq. (9) are as follows [26]:

$$
W=\left\{\begin{array}{l}
W_{1}=A_{1} \xi^{3}+B_{1} \xi^{2}+C_{1} \xi+D_{1} \\
W_{2}=A_{2} \cosh \xi \sin \xi+B_{2} \cosh \xi \cos \xi+C_{2} \sinh \xi \sin \xi+D_{2} \sinh \xi \cos \xi-\frac{F}{2} \sinh |\xi| \cos \xi+\frac{F}{2} \cosh \xi \sin |\xi| \\
W_{3}=A_{3} \xi^{3}+B_{3} \xi^{2}+C_{3} \xi+D_{3}
\end{array}\right.
$$

where $A_{i}, B_{i}, C_{i}$, and $D_{i}(i=1,2,3)$ are the 12 unknown constants to be determined. Because the lift-off points $\xi_{1}$ and $\xi_{2}$ are also unknown, there are total 14 unknowns to be determined. Therefore, 14 equations in total are needed to determine the above 14 unknowns. Four equations are from the boundary conditions, eight from the matching conditions and two from the constraint conditions.

For a free-free cylinder, the following four boundary conditions hold, which indicate the vanishing of the moment and shear force at the ends

$$
\begin{aligned}
& \frac{d^{2} w_{1}}{d x^{2}}\left(-L_{1}\right)=0, \frac{d^{3} w_{1}}{d x^{3}}\left(-L_{1}\right)=0, \quad \frac{d^{2} w_{3}}{d x^{2}}\left(L_{2}\right)=0, \\
& \frac{d^{3} w_{3}}{d x^{3}}\left(L_{2}\right)=0
\end{aligned}
$$

At the lift-off points $-x_{1}$ and $x_{2}$, the following eight matching conditions must be satisfied [26,27], which indicate the continuity of the displacement, slope, moment, and shear.

$$
\begin{gathered}
w_{1}\left(-x_{1}\right)=w_{2}\left(-x_{1}\right), \quad \frac{d w_{1}}{d x}\left(-x_{1}\right)=\frac{d w_{2}}{d x}\left(-x_{1}\right), \\
\frac{d^{2} w_{1}}{d x^{2}}\left(-x_{1}\right)=\frac{d^{2} w_{2}}{d x^{2}}\left(-x_{1}\right), \quad \frac{d^{3} w_{1}}{d x^{3}}\left(-x_{1}\right)=\frac{d w_{2}}{d x^{2}}\left(-x_{1}\right) \\
w_{2}\left(x_{2}\right)=w_{3}\left(x_{2}\right), \quad \frac{d w_{2}}{d x}\left(x_{2}\right)=\frac{d w_{3}}{d x}\left(x_{2}\right), \\
\frac{d^{2} w_{2}}{d x^{2}}\left(x_{2}\right)=\frac{d^{2} w_{3}}{d x^{2}}\left(x_{2}\right), \quad \frac{d^{3} w_{2}}{d x^{3}}\left(x_{2}\right)=\frac{d^{3} w_{3}}{d x^{3}}\left(x_{2}\right)
\end{gathered}
$$

These matching conditions, in essence, play the role of compatibility and equilibrium conditions, which physically guarantee that there is no fractures at those separation points (continuity of dis- placement and slope) and the continuity of moment and shear force. The boundary/matching conditions given by Weitsman [16] for an infinite beam are different from above ones and Gladwell [14] pointed out that Weitsman's boundary/matching conditions were problematic. Zhang and Murphy [26] showed that Weitsman's boundary/matching conditions were very good approximation for a very long beam. The same matching conditions as those in Eq. (A3) were also obtained by Ghatak et al. [20]. At the lift-off points, there are also two constraint conditions, which tell when the cylinder is in contact with the elastic medium [26]

$$
w_{1}\left(-x_{1}\right)=0, \quad w_{3}\left(x_{2}\right)=0
$$

Equations (A2)-(A4) offer 14 equations in total to solve the above 14 unknowns. Because of the unknown property of $\xi_{1}$ and $\xi_{2}$, the above 14 governing equations are highly nonlinear ones, which require the Newton-Rhapson method [45] to solve. Once the 14 unknowns are obtained, the displacement $W_{i}(i=1,2,3)$ is also obtained.

\section{References}

[1] Park, H. S., and Klein, P. A., 2008, "Surface Stress Effects on the Resonan Properties of Metal Nanowires: The Importance of Finite Deformation Kinematics and the Impact of the Residual Surface Stress," J. Mech. Phys. Solids, 56, pp. 3144-3166.

[2] Feng, G., Nix, W. D., Yoon, Y., and Lee, C. J., 2006, "A Study of the Mechanical Properties of Nanowires Using Nanoindentation,” J. Appl. Phys., 99, p. 074304 .

[3] Wang, Z. L., 2000, "Characterizing the Structure and Properties of Individua Wire-Like Nanoentities," Adv. Mater., 12, pp. 1295-1298.

[4] Jing, G. Y., Duan, H. L., Sun, X. M., Zhang, Z. S., Xu, J., Li, Y. D., Wang, J. X., and Yu, D. P., 2006, "Surface Effect on Elastic Properties of Silver Nanowire: Contact Atomic-Force Microscopy," Phys. Rev. B, 73, pp. 235409.

[5] Salvetat, J., Briggs, G. A. D., Bonard, J., Bacsa, R. R., Kulik, A. J., Stökli, T., Burham, N. A., and Forró, L., 1999, "Elastic and Shear Moduli of SingleWalled Carbon Nanotube Ropes," Phys. Rev. Lett., 82, pp. 944-947.

[6] Oliver, W. C., and Pharr, G. M., 1992, "An Improved Technique for Determining Hardness and Elastic Modulus Using Load and Displacement Sensing Indentation Experiments," J. Mater. Res., 7, pp. 1564-1583.

[7] Oliver, W. C., and Pharr, G. M., 2004, "Measurement of Hardness and Elastic 
Modulus by Instrumented Indentation: Advances in Understanding and Refinements to Methodology," J. Mater. Res., 19, pp. 3-20.

[8] Zhu, Y., Ke, C., and Espinosa, H. D., 2007, "Experimental Techniques for the Mechanical Characterization of One-Dimensional Nanostructures," Exp. Mech., 47, pp. 7-24.

[9] Castillo, J., and Barber, J. R., 1997, "Lateral Contact of Slender Prismatic Bodies," Proc. R. Soc. London, Ser. A, 453, pp. 2397-2412.

[10] Yu, M.-F., Kowalewski, T., and Ruoff, R. S., 2000, "Investigation of the Radial Deformability of Individual Carbon Nanotubes Under Controlled Indentation Force," Phys. Rev. Lett., 85, pp. 1456-1459.

[11] Keer, L. M., Dundurs, J., and Tsai, K. C., 1972, "Problems Involving a Receding Contact Between a Layer and a Half Space," ASME J. Appl. Mech., 39, pp. 1115-1120.

[12] Johnson, K. L., 1985, Contact Mechanics, Cambridge University Press, Cambridge, UK, Chaps. 4 and 5.

[13] Pu, S. L., and Hussain, M. A., 1970, "Note on the Unbonded Contact Between Plates and an Elastic Half Space," ASME J. Appl. Mech., 37, pp. 859-861.

[14] Gladwell, G. M., 1976, "On Some Unbonded Contact Problems in Plane Elasticity Theory," ASME J. Appl. Mech., 43, pp. 263-267.

[15] Weitsman, Y., 1969, "On the Unbonded Contact Between Plates and an Elastic Half Space," ASME J. Appl. Mech., 36, pp. 505-509.

[16] Weitsman, Y., 1970, "On Foundations That Reacts in Compression Only," ASME J. Appl. Mech., 37, pp. 1019-1030.

[17] Weitsman, Y., 1972, "A Tensionless Contact Between a Beam and an Elastic Half-Space," Int. J. Eng. Sci., 10, pp. 73-81.

[18] Ratwani, M., and Erdogan, F., 1973, "On the Plane Contact Problem for a Frictionless Elastic Layer," Int. J. Solids Struct., 9, pp. 921-936.

[19] Yang, W., Wang, H., and Huang, Y., 2005, "Abnormal Tribological Behavior of Multiwalled Nanotube Rafts. Part I: Aligned Rafts,” ASME J. Eng. Mater. Technol., 127, pp. 383-392.

[20] Ghatak, A., Mahadevan, L., and Chaudhury, M. K., 2005, "Measuring the Work of Adhesion Between a Soft Confined Film and a Flexible Plate," Langmuir, 21, pp. 1277-1281.

[21] Ghatak, A., Chaudhury, M. K., Shenoy, V., and Sharma, A., 2000, "Menicus Instability in a Thin Elastic Film," Phys. Rev. Lett., 85, pp. 4329-4332.

[22] Ghatak, A., Mahadevan, L., Chung, J. Y., Chaudhury, M. K., and Shenoy, V., 2004, "Peeling From a Biomimetically Patterned Thin Elastic Film," Proc. R. Soc. London, Ser. A, 460, pp. 2725-2735.

[23] Kendall, K., 1994, "Adhesion: Molecules and Mechanics," Science, 263, pp. 1720-1725.

[24] Chaudhury, M. K., Weaver, T., Hui, C. Y., and Kramer, E. J., 1996, “Adhesive Contact of Cylindrical Lens and a Flat Sheet," J. Appl. Phys., 80, pp. 30-37.

[25] Chen, S., and Gao, H., 2006, "Non-Slipping Adhesive Contact of an Elastic Cylinder on Stretched Substrate," Proc. R. Soc. London, Ser. A, 462, pp. 211-228.

[26] Zhang, Y., and Murphy, K. D., 2004, "Response of a Finite Beam in Contact With a Tensionless Foundation Under Symmetric and Asymmetric Loading," Int. J. Solids Struct., 41, pp. 6745-6758.

[27] Zhang, Y., 2008, "Tensionless Contact of a Finite Beam Resting on Reissner Foundation,” Int. J. Mech. Sci., 50, pp. 1035-1041.
[28] Kerr, A. D., 1964, "Elastic and Viscoelastic Foundation Models," ASME J. Appl. Mech., 31, pp. 491-498.

[29] Giannakopoulos, A. E., 2006, "Elastic and Viscoelastic Indentation of Flat Surfaces by Pyramid Indentors," J. Mech. Phys. Solids, 54, pp. 1305-1332.

[30] Yang, F., and Cheng, Y. T., 2009, "Revisit of the Two-Dimensional Indentation Deformation of an Elastic Half-Space," J. Mater. Res., 24, pp. 1976-1982.

[31] Biot, M. A., 1937, "Bending of an Infinite Beam on an Elastic Foundation," ASME J. Appl. Mech., 4, pp. 1-7.

[32] Nayfeh, A. H., and Mook, D. T., 1979, Nonlinear Oscillations, Wiley, New York, Chap. 2.

[33] Jiang, H., Sun, Y., Rogers, J. A., and Huang, Y., 2008, "Post-Buckling Analysis for the Precisely Controlled Buckling of Thin Film Encapsulated by Elastomeric Substrate," Int. J. Solids Struct., 45, pp. 2014-2023.

[34] Xiao, J., Jiang, H., Khang, D.-Y., Wu, J., Huang, Y., and Rogers, J. A., 2008, "Mechanics of Buckled Carbon Nanotubes on Elastomeric Substrate," J. Appl. Phys., 104, p. 033543.

[35] Xiao, J., Ryu, S., Huang, Y., Hwang, K., Paik, U., and Rogers, J., 2010, "Mechanics of Nanowire/Nanotubes In-Surface Buckling on Elastomeric Substrate," Nanotechnology, 21, p. 085708.

[36] Khang, D., Xiao, J., Kocabas, C., MacLaren, S., Banks, T., Jiang, H., Huang, Y., and Rogers, J., 2008, "Molecular Scale Buckling Mechanics in Individual Aligned Single-Wall Carbon Nanotubes on Elastomeric Substrate," Nano Lett. 8, pp. $124-130$

[37] Miller, R. E., and Shenoy, V. B., 2000, "Size-Dependent Elastic Properties of Nanosized Structural Elements," Nanotechnology, 11, pp. 139-147.

[38] Giri, M., Bousfield, D. B., and Unertl, W. N., 2001, "Dynamic Contacts on Viscoelastic Film: Work of Adhesion," Langmuir, 17, pp. 2973-2981.

[39] Sun, Y., Akhremitchev, B., and Walker, G. C., 2004, "Using the Adhesive Interaction Between Atomic Force Microscopy Tips and Polymer Surfaces to Measure the Elastic Modulus of Compliant Sample," Langmuir, 20, pp. 5837 5845 .

[40] Tabor, D., 1977, "Surface Force and Surface Interactions," J. Colloid Interface Sci., 58, pp. 2-13.

[41] Johnson, K. L., and Greenwood, J. A., 1997, "An Adhesion Map for the Contact of Elastic Spheres," J. Colloid Interface Sci., 192, pp. 326-333.

[42] Zhao, Y.-P., Wang, L. S., and Wang, T. X., 2003, "Mechanics of Adhesion in MEMS-A Review," J. Adhes. Sci. Technol., 17, pp. 519-546.

[43] Zhang, Y., 2008, "Transitions Between Different Contact Models," J. Adhes. Sci. Technol., 22, pp. 699-715.

[44] Bathe, K. J., 1996, Finite Element Procedures, Prentice-Hall, Englewood Cliffs, NJ, Chaps. 5 and 6.

[45] Press, W. H., Teukolsky, S. A., Vetterling, W. T., and Flannery, B. P., 1992, Numerical Recipes in Fortran, 2nd ed., Cambridge University Press, Cambridge, UK, Chap. 9.

[46] Everall, P. R., and Hunt, G. W., 2000, "Mode Jumping in the Buckling of Struts and Plates: A Comparative Study," Int. J. Non-Linear Mech., 35, pp. $1067-1079$.

[47] Zhang, Y., and Murphy, K., 2005, "Secondary Buckling and Tertiary States of a Beam on a Non-Linear Elastic Foundation," Int. J. Non-Linear Mech., 40, pp. 795-805. 